\title{
A computationally effective predictor-corrector method for simulating fractional order dynamical control system
}

$$
\text { C. Yang* } \quad \text { F. Liu* }{ }^{*}
$$

(Received 14 October 2005; revised 24 July 2006)

\begin{abstract}
Multi-order fractional differential equations are applied to fractional order dynamical controlled systems. The multi-order fractional differential equation is transferred into a system of fractional order differential equations. A new computationally effective fractional predictorcorrector method is proposed for simulating the fractional order systems and controllers. A detailed error analysis is derived. Finally, we give some numerical examples.
\end{abstract}

*School of Mathematical Sciences, Xiamen University, Xiamen 361005, China. mailto:morningsail@163.com

†School of Mathematical Sciences, Queensland University of Technology, Queensland 4001, Australia. mailto:f.liu@qut.edu.au.

See http://anziamj.austms.org.au/V47EMAC2005/Yang for this article, (C) Austral. Mathematical Soc. 2006. Published August 1, 2006. ISSN 1446-8735 


\section{Contents}

1 Introduction

C169

2 Basic ideas and lemmas

C172

3 A fractional predictor-corrector technique

C174

4 Error analysis for the fractional predictor-corrector method C176

5 Numerical Results

C179

6 Conclusions

C182

References

C183

\section{Introduction}

The Fractional Calculus is, simultaneously, a new and old research issue. This contradiction comes from the fact that most scientists are not aware of its existence and, often, discover that this 'new' tool provides fruitful perspectives in their studies. For three centuries the theory of fractional derivatives developed mainly as a pure theoretical field of mathematics useful only for mathematicians [1]. However, in the last few decades many have pointed out that derivatives and integrals of non-integer order are very suitable for the description of the properties of various real materials. It has been shown that new fractional order models are more adequate than previously used integer order models [1]. In the past two decades, fractional order control systems attracted the attention of many researchers lately [2]. However, because of the absence of appropriate mathematical methods, fractional order dynamical systems were studied only marginally in theory and practice of control systems. 


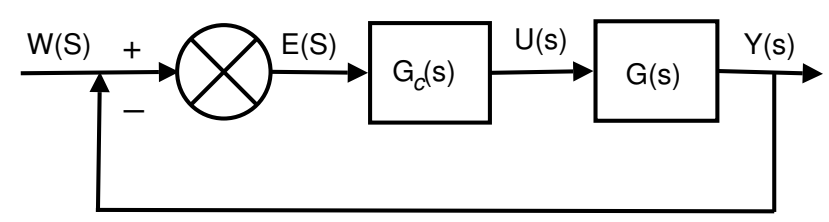

FiguRE 1: Simple unity-feedback control system

Let us consider the simple unity-feedback control system [1] shown in Figure 1, where $G(s)$ is the transfer function of the controlled system, $G_{c}(s)$ is the transfer of the controller, $W(s)$ is an input, $E(s)$ is an error, $U(s)$ is the controller's output, and $Y(s)$ is the system's output. Contrary to the traditional model, we consider transfer functions of arbitrary real order. Such systems are called fractional order control systems, which are better described by fractional order mathematical models.

Now consider the fractional order transfer function (FOTF) [1]:

$$
G_{n}(s)=\frac{1}{a_{n} s^{\alpha_{n}}+a_{n-1} s^{\alpha_{n-1}}+\cdots+a_{1} s^{\alpha_{1}}+a_{0} s^{\alpha_{0}}},
$$

where $\alpha_{n}>\alpha_{n-1}>\cdots>\alpha_{1}>\alpha_{0} \geq 0,0<\alpha_{n}-\alpha_{n-1}<1$, and $\alpha_{k}(k=$ $0,1, \ldots, n)$ are arbitrary real numbers. In the time domain, the FOTF (1) corresponds to the $(n+1)$-term fractional order differential equation

$$
a_{n} D^{\alpha_{n}} y(t)+a_{n-1} D^{\alpha_{n-1}} y(t)+\cdots+a_{1} D^{\alpha_{1}} y(t)+a_{0} D^{\alpha_{0}} y(t)=u(t),
$$

where $D^{\alpha} y={ }_{0}^{C} D_{t}^{\alpha} y=J^{m-\alpha} y^{(m)}(t)$ is the Caputo fractional derivatives (in time) of order $\alpha$ :

$$
{ }_{0}^{C} D_{t}^{\alpha} y(t)= \begin{cases}\frac{1}{\Gamma(m-\alpha)} \int_{0}^{t} \frac{y^{(m)}(\tau)}{(t-\tau)^{\alpha+1-m}} d \tau, & 0 \leq m-1<\alpha<m, \\ \frac{d^{m} y(t)}{d t^{m}}, & m \in \mathbb{N},\end{cases}
$$

where $m$ is the integer defined by the relation $m-1<\alpha<m$, and $J^{q}$ is the 
fractional integral operator

$$
J^{q} y(t)=\frac{1}{\Gamma(q)} \int_{0}^{t}(t-\tau)^{q-1} y(\tau) d \tau .
$$

Numerical solution of a fractional order differential equation is difficult to obtain accurately using standard discretization methods. Hu and Liu [3] considered a four term fractional differential equation corresponding to a fractional order controlled system. The existence and uniqueness of the solution of this model are given, and they proposed three numerical methods for the fractional order control systems. Shen and Liu considered [4] a fractional order Bagley-Torvik equation, and proved the existence and uniqueness of solution. The analytical solution of a fractional order Bagley-Torvik equation is also derived by the corresponding Green's function. Using the relationship between the Riemann-Liouville definition and the Gronwald-Letnikov definition, Shen and Liu proposed a computationally effective method for the fractional order Bagley-Torvik Equation. Diethelm et al. [5] proposed some techniques for solving fractional order ordinary differential equations and analysed errors. Lin and Liu [6] also proposed a fractional order numerical method for the fractional relaxation equation, and the consistence, convergence and stability of this method is proven. Liu et al. [7] proposed some efficient fractional numerical methods for solving fractional partial differential equation. A special multi-order fractional differential equation was also considered by Miller and Ross [8]. The multi-order fractional differential equations were written as an equivalent system of fractional order differential equations. However, numerical methods for solving fractional order differential systems are limited. Therefore, a new numerical strategy is important in solving these fractional order systems. We propose a new technique for solving fractional order differential equation systems and are apply the technique to simulate a fractional order control system.

Some basic ideas and lemmas are presented in Section 2. A computationally effective fractional predictor-corrector method is proposed in Section 3. 
A detailed error analysis is derived in Section 4. Finally, Section 5 gives some numerical results.

\section{Basic ideas and lemmas}

We are concerned with providing good quality methods for the solution of multi-order fractional differential equations of the general form

$$
\begin{aligned}
& a_{n} D^{\alpha_{n}} y(t)+a_{n-1} D^{\alpha_{n-1}} y(t)+\cdots+a_{1} D^{\alpha_{1}} y(t)+a_{0} D^{\alpha_{0}} y(t)=f(t), \\
& y^{k}(0)=y_{0}^{(k)}, \quad k=0,1, \ldots,\left\lceil\alpha_{n}\right\rceil
\end{aligned}
$$

where $\alpha_{n}>\alpha_{n-1}>\cdots>\alpha_{1}>\alpha_{0} \geq 0,0<\alpha_{n}-\alpha_{n-1}<1, \alpha_{k}(k=$ $0,1, \ldots, n)$ are arbitrary real numbers, $a_{k}(k=0,1, \ldots, n)$ are arbitrary constants.

The basic analytical results on existence and uniqueness of solutions to fractional differential equations are given in $[1,8]$. Miller and Ross [8] gave a method for calculating the analytic solution to a multi-term fractional differential equation of the form

$$
a_{n} D^{n \alpha} y(t)+a_{n-1} D^{(n-1) \alpha} y(t)+\cdots+a_{1} D^{\alpha} y(t)+a_{0} D^{0} y(t)=0,
$$

where $\alpha=1 / M, M \in \mathbb{N}$. They interpret the fractional operator $D^{i \alpha}$ as $D^{\alpha}$ applied $i$ times. Under this interpretation they describe the multi-term fractional differential equation as a sequential fractional differential equation.

For the sake of simplicity we assume that $\alpha_{i}=i / 2=m_{i} \in \mathbb{N}$, if $i$ is an even number; $(i-1) / 2<\alpha_{i}<(i+1) / 2$, if $i$ is an odd number; that is, there is at most one non-integer order derivative between successive integer orders.

Lemma 1 Let $y \in C^{k}[0, T]$ for some $T>0$ and some $k \in \mathbb{N}$, and let $\beta \notin \mathbb{N}$ such that $0<\beta<k$, then ${ }_{a}^{C} D_{t}^{\beta} y(0)=0$. 
Proof: See [9].

Lemma 2 The differentiation operators ${ }_{a}^{C} D_{t}^{\alpha} f(t)$ and ${ }_{a}^{C} D_{t}^{m} f(t)$ satisfy the interchange rule:

$$
\begin{aligned}
& { }_{0}^{C} D_{t}^{\alpha}\left({ }_{0}^{C} D_{t}^{m} f(t)\right)={ }_{0}^{C} D_{t}^{m}\left({ }_{0}^{C} D_{t}^{\alpha} f(t)\right)={ }_{0}^{C} D_{t}^{\alpha+m} f(t), \\
& f^{(s)}(0)=0, \quad s=n, n+1, \ldots, m, \quad m=0,1, \ldots, n-1<\alpha<n .
\end{aligned}
$$

Proof: See [1].

Let $y \in C^{\left\lceil\alpha_{n}\right\rceil}[0, T]$ for some $T>0$. Using Lemma 1, we have

$$
{ }_{0}^{C} D_{t}^{\alpha_{2 i}-\alpha_{2 i-1} C}{ }_{0} D_{t}^{\alpha_{2 i-1}} y(t)={ }_{0}^{C} D_{t}^{\alpha_{2 i}} y, \quad{ }_{0}^{C} D_{t}^{\alpha_{2 i+1}-\alpha_{2 i} C} D_{t}^{\alpha_{2 i}} y(t)={ }_{0}^{C} D_{t}^{\alpha_{2 i+1}} y .
$$

Now we rewrite the multi-order fractional differential equations (5) in the form of a system of fractional order differential equations:

$$
\left\{\begin{array}{l}
{ }_{0}^{C} D_{t}^{\beta_{1}} x_{1}(t)={ }_{0}^{C} D_{t}^{\alpha_{1}} x_{1}(t)=x_{2}(t), \\
\vdots \\
{ }_{0}^{C} D_{t}^{\beta_{n-1}} x_{n-1}(t)={ }_{0}^{C} D_{t}^{\alpha_{n-1}-\alpha_{n-2}} x_{n-1}(t)=x_{n}(t), \\
{ }_{0}^{C} D_{t}^{\beta_{n}} x_{n}(t)={ }_{0}^{C} D_{t}^{\alpha_{n}-\alpha_{n-1}} x_{n}(t) \\
=\frac{1}{a_{n}}\left[f(t)-a_{0} x_{1}-a_{1} x_{2}-\cdots-a_{n-1} x_{n}\right],
\end{array}\right.
$$

with initial equations

$$
\begin{aligned}
& x_{1}(0)=x_{0}^{(1)}=y_{0}^{(0)}, \quad x_{2}(0)=x_{0}^{(2)}=0, \quad \ldots, \\
& x_{i}(0)=x_{0}^{(i)}=\left\{\begin{array}{lll}
y_{0}^{(k)}, & \text { if } i=2 k+1, & i \leq n . \\
0, & \text { if } i=2 k,
\end{array}\right.
\end{aligned}
$$

Using Lemma 1 and Lemma 2, we obtain the following theorem: 
Theorem 3 The multi-order fractional differential equations (5) is equivalent to the system of equations (9) with the initial conditions (10).

\section{A fractional predictor-corrector technique}

In this section, numerical technique for simulate fractional order control systems (1) are presented. Firstly, the fractional order differential systems is decoupled, which is equivalent to solving

$$
\left\{\begin{array}{cc}
{ }_{0}^{C} D_{t}^{\beta_{1}} x_{1}(t) & =g_{1}\left(t, x_{1}\right), \\
\vdots & \\
{ }_{0}^{C} D_{t}^{\beta_{n}} x_{n}(t) & =g_{n}\left(t, x_{n}\right) .
\end{array}\right.
$$

Secondly, we consider a computationally effective fractional predictorcorrector method for solving the following initial-value problem:

$$
{ }_{0}^{C} D_{t}^{\beta_{i}} x_{i}(t)=g_{i}\left(t, x_{i}\right), \quad x_{i}(0)=x_{0}^{(i)}, \quad i=1,2, \ldots, n,
$$

where $0<\beta_{i}<1$.

It is well known that the initial-value problem (12) is equivalent to the Volterra integral equation

$$
x_{i}(t)=x_{0}^{(i)}+\frac{1}{\Gamma\left(\beta_{i}\right)} \int_{0}^{t}(t-\tau)^{\beta_{i}-1}\left[g_{i}\left(\tau, x_{i}(\tau)\right)\right] d \tau .
$$

For the sake of simplicity, we assume that we are working on a uniform grid $t_{j}=j \tau, j=0,1, \ldots, M \tau=T$.

The issue of stability is very important when implementing the method on a computer in finite precision arithmetic because we must take into account effects introduced by rounding errors. It is known that the classical AdamsBashforth-Moulton method for first order ordinary differential equations is 
a reasonable and practically useful compromise in the sense that its stability properties allow for a safe application to mildly stiff equations without undue propagation of rounding error, whereas the implementation does not require extremely time consuming elements [10]. Thus, a fractional AdamsBashforth method and a fractional Adams-Moulton method are chosen as our predictor and corrector formulas.

The predictor $x_{i, k+1}^{P}$ is determined by the fractional Adams-Bashforth method $[5,11]$ :

$$
x_{i, k+1}^{P}=x_{0}^{(i)}+\frac{1}{\Gamma(\alpha)} \sum_{j=0}^{k} b_{j, k+1}^{\beta_{j}} g_{i}\left(t_{j}, x_{i, j}\right)
$$

where

$$
b_{j, k+1}^{\beta}=\frac{\tau^{\beta}}{\beta}\left[(k+1-j)^{\beta}-(k-j)^{\beta}\right] .
$$

The fractional Adams-Moulton method determines the corrector formula [5, 11]:

$$
x_{i, k+1}=x_{0}^{(i)}+\frac{1}{\Gamma\left(\beta_{i}\right)}\left(\sum_{j=0}^{k} a_{j, k+1}^{\beta_{i}} g_{i}\left(t_{j}, x_{i, j}\right)+a_{k+1, k+1}^{\beta_{i}} g_{i}\left(t_{k+1}, x_{i, k+1}^{P}\right)\right),
$$

where

$$
a_{j, k+1}^{\beta}=\frac{\tau^{\beta}}{\beta(\beta+1)}\left\{\begin{array}{cr}
k^{\beta+1}-(k-\beta)(k+1)^{\beta}, & j=0, \\
(k-j+2)^{\beta+1}+(k-j)^{\beta+1} & \\
-2(k-j+1)^{\beta+1}, & 1 \leq j \leq k, \\
1, & j=k+1 .
\end{array}\right.
$$

Therefore, we obtain the following new fractional predictor-corrector method for solving the fractional order differential systems (9). 
Fractional Predictor formulas:

$$
\begin{aligned}
& x_{i, k+1}^{P}=x_{0}^{(i)}+\frac{1}{\Gamma\left(\beta_{i}\right)} \sum_{j=0}^{k} b_{j, k+1}^{\beta_{i}} x_{i+1, j}, \quad i=1,2, \ldots, n-1, \\
& x_{n, k+1}^{P}=x_{0}^{(i)}+\frac{1}{\Gamma\left(\beta_{n}\right)} \sum_{j=0}^{k} b_{j, k+1}^{\beta_{n}} \frac{1}{a_{n}}\left[u\left(t_{j}\right)-a_{0} x_{1, j}-\cdots-a_{n-1} x_{n, j}\right]
\end{aligned}
$$

Fractional Corrector formulas:

$$
\begin{aligned}
x_{i, k+1}= & x_{0}^{(i)}+\frac{1}{\Gamma\left(\beta_{i}\right)}\left(\sum_{j=0}^{k} a_{j, k+1}^{\beta_{i}} x_{i+1, j}+a_{k+1, k+1}^{\beta} x_{i+1, k+1}^{P}\right), \\
& i=1,2, \ldots, n-1, \\
x_{n, k+1}= & x_{0}^{(i)}+\frac{1}{\Gamma\left(\beta_{n}\right)}\left\{\sum_{j=0}^{k} a_{j, k+1}^{\beta_{n}} \frac{1}{a_{n}}\left[u\left(t_{j}\right)-a_{0} x_{1, j}-\cdots-a_{n-1} x_{n, j}\right]\right. \\
& \left.+a_{k+1, k+1}^{\beta_{n}} \frac{1}{a_{n}}\left[u\left(t_{k+1}\right)-a_{0} x_{1, k+1}^{P}-\cdots-a_{n-1} x_{n, k+1}^{P}\right]\right\}
\end{aligned}
$$

\section{Error analysis for the fractional predictor-corrector method}

In this section we present the theorems concerning the error of our fractional predictor-corrector method.

Lemma 4 Let $z \in C^{1}[0, T]$, then

$$
\left|\int_{0}^{t_{k+1}}\left(t_{k+1}-t\right)^{\beta-1} z(t) d t-\sum_{j=0}^{k} b_{j, k+1}^{\beta} z\left(t_{j}\right)\right| \leq \frac{1}{\beta}\left\|z^{\prime}\right\|_{\infty} t_{k+1}^{\beta} \tau .
$$


Proof: See $[5,11]$.

Lemma 5 If $z \in C^{2}[0, T]$, then there is a constant $C_{\beta}$ depending only on $\beta$ such that

$$
\left|\int_{0}^{t_{k+1}}\left(t_{k+1}-t\right)^{\beta-1} z(t) d t-\sum_{j=0}^{k+1} a_{j, k+1}^{\beta} z\left(t_{j}\right)\right| \leq C_{\beta}\left\|z^{\prime \prime}\right\|_{\infty} t_{k+1}^{\beta} \tau^{2} .
$$

Proof: See $[5,11]$.

Theorem 6 If ${ }_{0}^{C} D_{t}^{\beta_{i}} x_{i} \in C^{2}[0, T],(i=1,2, \ldots, n)$, then

$$
\max _{\substack{0 \leq j \leq M \\ 1 \leq i \leq n}}\left|x_{i}\left(t_{j}\right)-x_{i, j}\right|=O\left(h^{q}\right)
$$

where $q=1+\min _{1 \leq i \leq n} \beta_{i}$.

Proof: Using given condition ${ }_{0}^{C} D_{t}^{\beta_{i}} x_{i} \in C^{2}[0, T],(i=1,2, \ldots, n)$, Lemma 4 and Lemma 5, we have

$$
\begin{aligned}
& \left|\int_{0}^{t_{k+1}}\left(t_{k+1}-t\right)_{0}^{\beta_{i}-1 C} D_{t}^{\beta_{i}} x_{i}(t) d t-\sum_{j=0}^{k} b_{j, k+10}^{\beta} D_{t}^{\beta_{i}} x_{i}\left(t_{j}\right)\right| \leq C_{1} t_{k+1}^{\beta_{i}} \tau, \\
& \left|\int_{0}^{t_{k+1}}\left(t_{k+1}-t\right)_{0}^{\beta_{i}-1 C} D_{t}^{\beta_{i}} x_{i}(t) d t-\sum_{j=0}^{k+1} a_{j, k+10}^{\beta} D_{t}^{\beta_{i}} x_{i}\left(t_{j}\right)\right| \leq C_{2} t_{k+1}^{\beta_{i}} \tau^{2} .
\end{aligned}
$$

We show that, for sufficiently small $\tau=T / M$,

$$
\max _{\substack{0 \leq j \leq M \\ 1 \leq i \leq n}}\left|x_{i}\left(t_{j}\right)-x_{i, j}\right|=O\left(\tau^{q}\right)
$$


where $q=1+\min _{1 \leq i \leq n} \beta_{i}$. The proof will be based on mathematical induction. In view of the given initial condition, the induction basis $(j=0)$ is presupposed.

Now assume that (26) is true for $j=0,1, \ldots, k(k \leq M-1)$, that is,

$$
\max _{\substack{0 \leq j \leq M \\ 1 \leq j \leq n}}\left|x_{i}\left(t_{j}\right)-x_{i, j}\right|=O\left(\tau^{q}\right)
$$

We must then prove the inequality also holds for $j=k+1$. To do this, we first look at the error of the predictor solutions $x_{i, k+1}^{P}, i=1,2, \ldots, n$. By construction of the predictor formulas, using (24), assumption (27), and

$$
\sum_{j=0}^{k} b_{j, k+1}^{\beta_{i}}=\int_{0}^{t_{k+1}}\left(t_{k+1}-t\right)^{\beta_{i}-1} d t=\frac{1}{\beta_{i}} t_{k+1}^{\beta_{i}} \leq \frac{1}{\beta_{i}} T^{\beta_{i}}
$$

we find that

$$
\begin{aligned}
& \left|x_{i}\left(t_{k+1}\right)-x_{i, k+1}^{P}\right| \leq \frac{C_{1} T^{\beta_{i}}}{\Gamma\left(\beta_{i}\right)} \tau+\frac{C_{0} T^{\beta_{i}}}{\Gamma\left(\beta_{i}+1\right)} \tau^{q}, \quad i=1,2, \ldots, n-1, \\
& \left|x_{n}\left(t_{k+1}\right)-x_{n, k+1}^{P}\right| \leq \frac{C_{1} T^{\beta_{n}}}{\Gamma\left(\beta_{n}\right)} \tau+\frac{\bar{C}_{0} L T^{\beta_{n}}}{\Gamma\left(\beta_{n}+1\right)} \tau^{q}
\end{aligned}
$$

Now we begin the analysis of the corrector error. For $j=k+1$, arguing in a similar way to the above, by construction of the predictor formulas, using (25), assumption (27), and (28)-(29), we find that

$$
\begin{aligned}
& \left|x_{i}\left(t_{k+1}\right)-x_{i, k+1}\right| \\
\leq & \left(\frac{C_{2} T^{\beta_{i}}}{\Gamma\left(\beta_{i}\right)}+\frac{C_{0} T^{\beta_{i}}}{\Gamma\left(\beta_{i}+1\right)}+\frac{C_{1} T^{\beta_{i}}}{\Gamma\left(\beta_{i}\right) \Gamma\left(\beta_{i}+2\right)}+\frac{C_{0} T^{\beta_{i}}}{\Gamma\left(\beta_{i}+1\right) \Gamma\left(\beta_{i}+2\right)} \tau^{\beta_{i}}\right) \tau^{q} \\
\leq & C \tau^{q}, \quad i=1,2, \ldots, n-1 \\
& \left|x_{n}\left(t_{k+1}\right)-x_{n, k+1}\right|
\end{aligned}
$$




$$
\begin{aligned}
& \leq\left(\frac{C_{2} T^{\beta_{n}}}{\Gamma\left(\beta_{n}\right)}+\frac{C_{0} T^{\beta_{n}}}{\Gamma\left(\beta_{n}+1\right)}+\frac{C_{1} T^{\beta_{n}}}{\Gamma\left(\beta_{n}\right) \Gamma\left(\beta_{n}+2\right)}+\frac{\bar{C}_{0} T^{\beta_{n}}}{\Gamma\left(\beta_{n}+1\right) \Gamma\left(\beta_{n}+2\right)} \tau^{\beta_{n}}\right) \tau^{q} \\
& \leq C \tau^{q} .
\end{aligned}
$$

This completes the proof.

Therefore, we obtain not only an approximation for the solution $y(t)$ of the $n$ term fractional order differential equation, but also approximations for its (Caputo type) derivatives of order $\alpha_{i}(i=1,2, \ldots, n-1)$. Apart from this useful feature, the method has a rather simple structure that makes it very easy to implement.

\section{$5 \quad$ Numerical Results}

Consider a fractional order controlled system with transfer function $[1,2]$

$$
G_{4}(s)=\frac{1}{a_{4} s^{\alpha_{4}}+a_{3} s^{\alpha_{3}}+a_{2} s^{\alpha_{2}}+a_{1} s^{\alpha_{1}}+a_{0}},
$$

where we take $0<\alpha_{1}<1.0, \alpha_{2}=1.0,1.0<\alpha_{3}<2.0, \alpha_{4}=2.0, a_{0}=1$, $a_{1}=0, a_{2}=0, a_{3}=0.5, a_{4}=1$. The fractional order transfer function (32) corresponds in the time domain to the five term fractional order differential equation

$$
a_{4} D^{\alpha_{4}} y(t)+a_{3} D^{\alpha_{4}}+a_{2} D^{\alpha_{2}} y(t)+a_{1} D^{\alpha_{1}} y(t)+a_{0} y(t)=u(t),
$$

with the initial conditions

$$
y(0)=y_{0}^{(0)}=0, y^{\prime}(0)=y_{0}^{(1)}=1 .
$$

Now rewrite the multi-order fractional differential equations (32) as a 


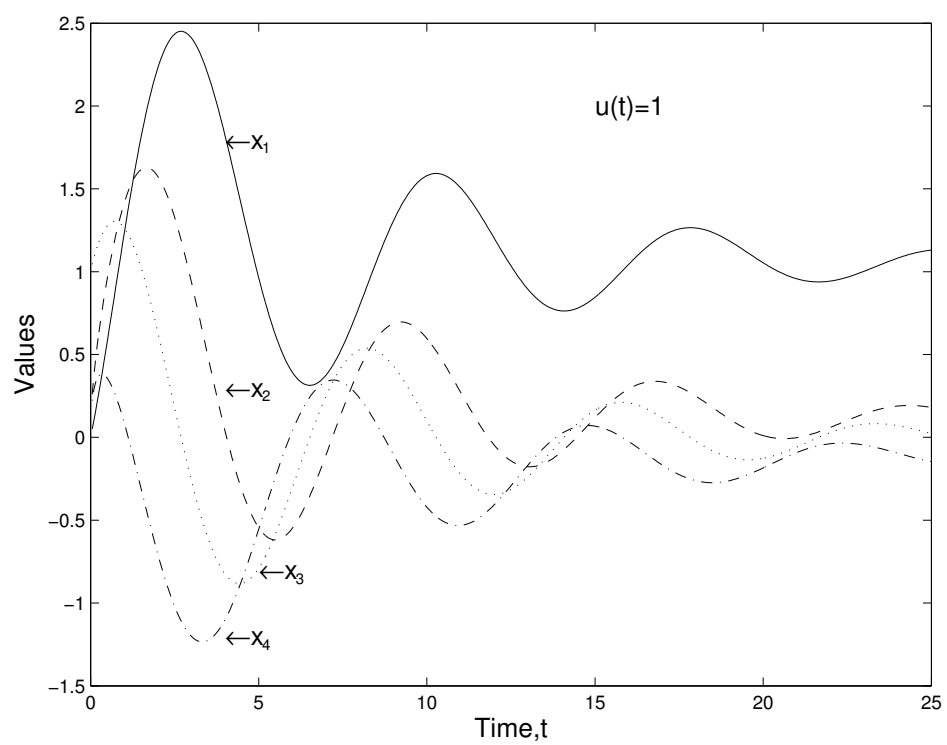

FiguRE 2: Unit-step response of the fractional order system with $u(t)=1$.

system of fractional order differential equations:

$$
\left\{\begin{array}{l}
{ }_{0}^{C} D_{t}^{\beta_{1}} x_{1}(t)={ }_{0}^{C} D_{t}^{\alpha_{1}} x_{1}(t)=x_{2}(t), \\
{ }_{0}^{C} D_{t}^{\beta_{2}} x_{2}(t)={ }_{0}^{C} D_{t}^{\alpha_{2}-\alpha_{1}} x_{2}(t)=x_{3}(t), \\
{ }_{0}^{C} D_{t}^{\beta_{3}} x_{3}(t)={ }_{0}^{C} D_{t}^{\alpha_{3}-\alpha_{2}} x_{3}(t)=x_{4}(t), \\
{ }_{0}^{C} D_{t}^{\beta_{4}} x_{4}(t)={ }_{0}^{C} D_{t}^{\alpha_{4}-\alpha_{3}} x_{4}(t)=\frac{1}{a_{4}}\left[u(t)-a_{0} x_{1}-a_{1} x_{2}-a_{2} x_{3}-a_{3} x_{4}\right],
\end{array}\right.
$$

with initial conditions

$$
x_{1}(0)=y_{0}^{(0)}=0, \quad x_{2}(0)=0, \quad x_{3}(0)=y_{0}^{(1)}=1, \quad x_{4}(0)=0 .
$$

Example 1. We take $\alpha_{1}=0.5, \alpha_{2}=1.0, \alpha_{3}=1.5, \alpha_{4}=2.0$. 


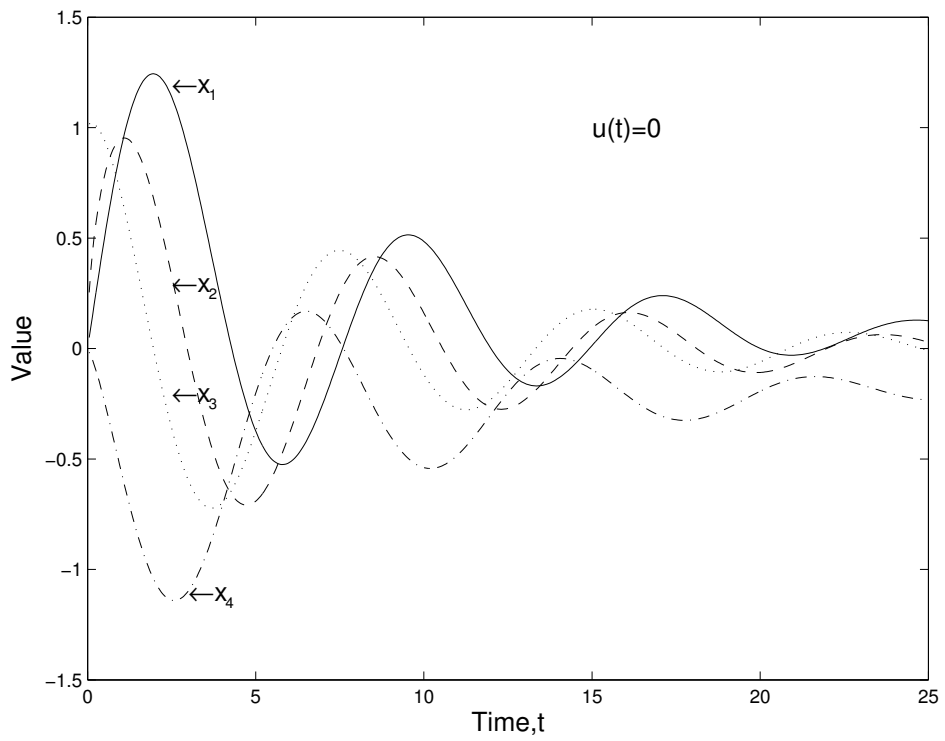

FiguRE 3: Unit-step response of the fractional order system with $u(t)=0$.

Figures 2 and 3 show the simulation results with $u(t)=1$ and $u(t)=0$, respectively. The system exhibits behaviors of the solution and its derivatives of order 0.5,1,1.5. From Figures 2 and 3, see that the computed results are in excellent agreement with those of Wang et al. [12].

Example 2. We take $\alpha_{1}=0.5, \alpha_{2}=1.0,1.1 \leq \alpha_{3} \leq 1.9, \alpha_{4}=2.0$.

Figure 4 displays the effect of $1.1 \leq \alpha_{3} \leq 1.9$ (that is, $0.1 \leq \beta_{3} \leq 0.9$ ) using predictor-corrector method for the fractional order control system with $u(t)=1$. From Figure 4 shows that when $\beta_{3}$ increases from 0.1 to 0.9 , the oscillation of solution also increases.

Figures 2-4 show that the fractional predictor-corrector method provides a computationally effective method for simulating the behavior of the solution of a fractional order control system. 


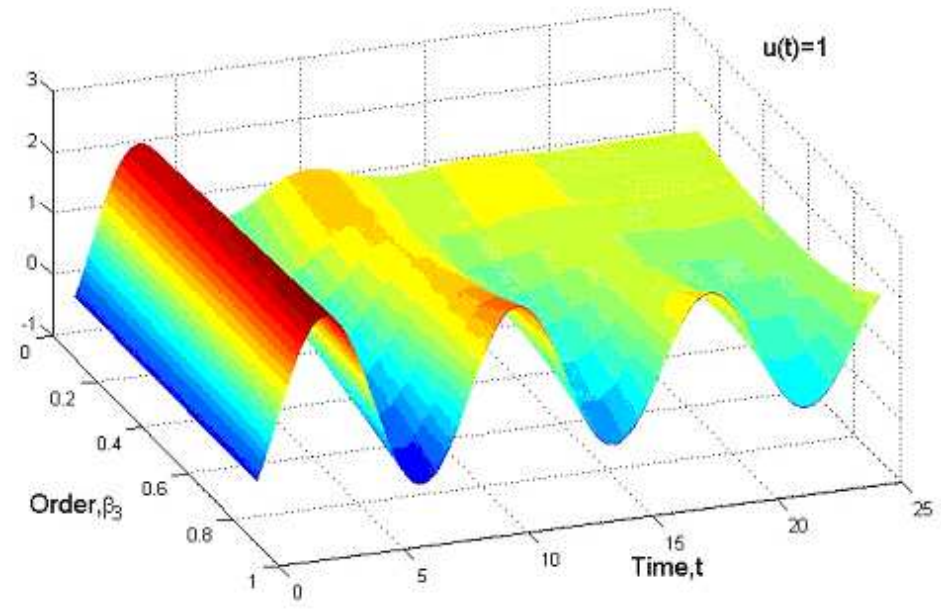

FiguRE 4: Solution of fractional order system for $0.1<\beta_{3}<0.9, u(t)=1$.

\section{Conclusions}

A fractional predictor-corrector method for simulating the fractional order control systems has been described and demonstrated. This method provides a computationally effective method for simulating the behavior of the solution of a fractional control system. A detailed error analysis is discussed. This numerical technique can be applied to simulate other fractional order differential system.

Acknowledgments: This research has been supported by the National Natural Science Foundation of China grant 10271098. 


\section{References}

[1] Podlubny, I., Fractional differential equations. New York: Academic Press, 1999. http://www.tuke.sk/podlubny/fde.html C169, C170, C172, C173, C179

[2] Petras, I. and Dorcak, L., Fractional-order control systems: modeling and simulation, fractional calculus and applied analysis, 6, 2003, 205-232.

http://www. diogenes.bg/fcaa/volume6/fcaa62/apetrased.pdf C169, C179

[3] Hu Y. and Liu F., Numerical methods for a fractional-order control system, Journal of Xiamen University (NATURAL Science), 44, 2005, 313-317. URL C171

[4] Shen S., Liu F., A computational effective method for fractional order Bagley-Torvik equation, Journal of Xiamen University (NATURAL Science), 45, 2004, 306-311. URL C171

[5] Diethelm K., Ford N. J., and Freed Alen D., Detailed error analysis for a fractional adams method[M], Numerical Algorithms, Kluwer Academic Publishers, 2004, 31-52. C171, C175, C177

[6] Lin R., Liu F., Analysis of fractional-order numerical method for the fractional relaxation equation, Computational Mechanics, Tsinghua University and Springer-Verlag, 2004, R-362. http://dx.doi.org/10.1016/j.na.2005.12.027 C171

[7] Liu F., Anh V., Turner I., Numerical solution of the space fractional Fokker-Planck equation, J. Comp. and Appl. Math., 166, 2004, 209-219. http://dx.doi.org/10.1016/j.cam.2003.09.028 C171 
[8] Miler K. S., Ross B., An introduction to the fractional calculus and fractional differential equations, 209-217, John Wiley, 1993. C171, C172

[9] Diethelm K., Ford N. J., Numerical solution of the Bagley-Torvik equation, BIT 42, 2002, 490-507. C173

[10] Hairer E., Wanner G. Solving ordinary differential equations II: stiff and differential-algebraic problems. Springer, Berlin. 1991. URL C175

[11] Yang C., Liu F., A fractional predictor-corrector method of the fractional relaxation-oscillation equation, Journal of Xiamen University, 44, 2005, 761-765. URL C175, C177

[12] Wang Z., Cao G., Two system modeling methods using fractional calculus, J. System Simulation, 16, 2004, 810-812. URL C181 